International Journal of Quantum Information

Vol. 6, No. 2 (2008)

\title{
OUTLINE OF THE SECOQC QUANTUM-KEY-DISTRIBUTION NETWORK IN VIENNA
}

\author{
A. POPPE, M. PEEV and O. MAURHART \\ on behalf of the Integrated European Project SECOQC - Development of a \\ Global Network for Secure Communication Based on Quantum Cryptography* \\ andreas.poppe@arcs.ac.at
}

\begin{abstract}
A Quantum Key Distribution (QKD) network is currently implemented in Vienna by integrating seven QKD-Link devices that connect five subsidiaries of SIEMENS Austria. We give an architectural overview of the network and present the enabling QKDtechnologies, as well as the novel QKD network protocols.
\end{abstract}

Keywords: Quantum key distribution; quantum cryptography; quantum network.

\section{Motivation}

The rapid progress in theory $1 / 2 \mid 3$ and experiment ${ }^{4}$ of QKD techniques has been reflected by a number of successful demonstrations in the last few years. Many groups all over the world have put forward QKD setups operating in the standard point-to-point modus, thus realizing what we denote as QKD-Links [Fig. 1(a)]. Initial attempts to assemble QKD based networks have also been launched $\underline{5}$

A number of research spin-offs (IdQuantique, MagiQ and SmartQuantum) offer QKD technology but also some internationally operating companies (e.g. Toshiba, Thales, HP and NEC) develop QKD setups aiming at a market realization in near future. The secure communication solutions pursued are typically based on dedicated high-end symmetric-encryption devices with frequent key change, whereby fresh key is constantly generated by QKD devices. The resulting QKD-LinkEncryptors (with encryption rates potentially up to $10 \mathrm{~Gb}$ per second) offer comparable functionality to existing products but feature higher level of security. Broad proliferation of QKD technology is hindered however by a number of road blocks revolving typically around: the point-to-point paradigm and correspondingly the quadratic scaling of the initial secrets with the number of users, the question of integrability in existing networks, the high price of QKD devices, but also around issues like missing standards. As a result, QKD appears to be forced into a narrow niche market.

The EU funded FP6 project SECOQC - Development of a Global Network for

${ }^{*}$ For affiliations of the SECOQC partners see http://www.secoqc.net/html/partners.html 
Secure Communication based on Quantum Cryptography aims to work against these limitations. A standardization initiative and a dedicated effort on optimization of single-photon-detection (a major technological challenge for QKD resulting in a heavy cost factor) are part of this project. The main goal, however, is taking in operation and testing an integrated secrets' distribution network, designed as a Quantum Back Bone network (QBB) deployed for test purposes in the city of Viennab. Thereby heterogenic QKD-systems by different SECOQC partners are connected with node modules, which overtake the network functionalities. The QBB, deployed on a typical telecommunication metropolitan area network (MAN) is an important step towards demonstrating the feasibility of fully networked multipoint to multi-point quantum key distribution. A target market of networked secure communication solutions for organizations with distributed facilities such as governmental institutions, companies and banks is addressed.
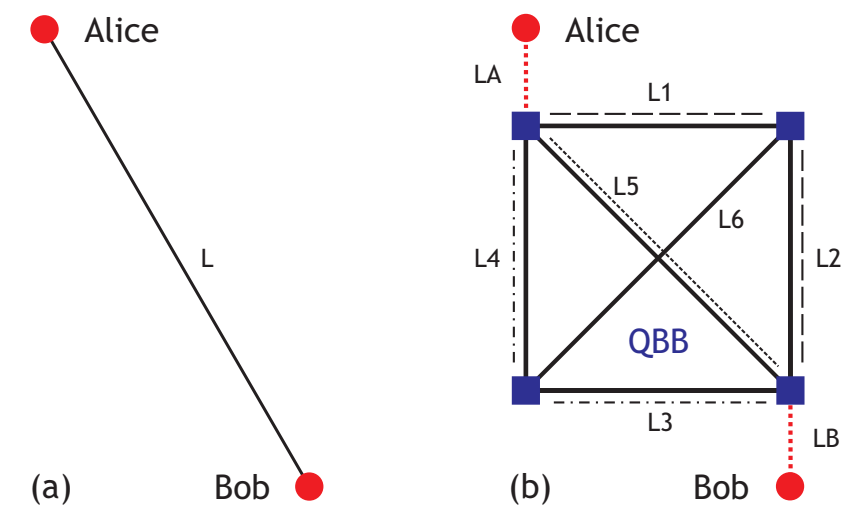

Fig. 1. (Color online). Alice and Bob want to share a secret key. (a) Point-to point QKD-Link L: A typical solution nowadays. (b) Quantum network: The QKD-Links LA and LB are used to access the $\mathrm{QBB}$, the nodes of the $\mathrm{QBB}$ being represented by squares (in blue) and the corresponding QKD-Links being denoted by L1 through L6. All QKD-Links provide unconditionally secure channels. An end-to-end secure channel from Alice to Bob along the paths LA-L5-LB, LA-L1L2-LB and LA-L3-L4-LB allows secure transmission of a secret key.

\section{Advantages of Using QKD-Networks}

The underlying idea is to build up a network for distributing secrets out of single point-to-point QKD-Links. The corresponding QKD-Link end points (i.e. the QKD devices) are situated in network nodes. These nodes are secure sites, in which one or more QKD devices are located together with a central node module, dedicated to processing, storage and communication. These node modules are the network

\footnotetext{
${ }^{a}$ See http://www. secoqc.net. The work presented here originates from the combined efforts of 41 project partners working together in various subgroups.

${ }^{\mathrm{b}}$ To be demonstrated in September 2008.
} 
agents taking over (in the SECOQC approach) full control of classical communication channels, the management of the generated secret keys, their informationtheoretical secure transmission from node to node on a hop-by-hop manner, $\frac{6}{6}$ and issues like finding paths to distant nodes and ensuring the synchrony of secret key provision to key-consuming applications across the network.

A QKD-network, following this design, is then an infrastructure for distributing the secret keys between nodes on a many-to-many basis over potentially unlimited distances. Under the assumption that the nodes can be trusted, it allows to utilize the information-theoretic security of quantum key distribution and achieve unconditional key distribution across the network. It should be underlined that while other approaches to designing quantum key distribution have been put forward (see e.g. Ref. 6 for a discussion) the trusted repeater architecture, adopted by SECOQC, 7 is the only currently feasible one that allows both going beyond the point-to-point and the limited distance constraints of standard QKD.

Figure 1(b) shows an universal building block: a rectangle with diagonals that is formed by four QBB-nodes (squares) connected together by six links. Additionally, each QBB node may act as a possible entry for end-users to be connected to the QBB by a single QKD-Link. This quantum access network (QAN) is shown in Fig. 1(b) for an example of two users, Alice and Bob (circles).

In what follows, we list the main advantages of QKD-networks over single QKDLinks:

- To include an additional end-user into the network, only a single QKD-Link is needed for connecting him to the closest node of the QBB. Then all other users already attached at the QBB are potential partners and can share a key with him.

- For a high number of end-users, the scaling of a QKD-network comprising a QBB and QANs is strongly favorable. For single point-to-point connections the number of needed QKD-Links increases as $\frac{N(N-1)}{2}$ for $N$ users. The presented network grows only linearly with $N$, as this is the number of the needed QAN-Links.

- In a metropolitan area, setting a direct fiber link between Alice and Bob would typically be far longer than the corresponding distances connecting both endusers to the QBB. Due to this lower loss of the access links and the potentially high supply of secure key through the QBB, the overall key rate will be higher using a QKD-network.

- Within the QBB, links can be cascaded in series to connect users at longer distances and in parallel to obtain higher values of secure key rate. Fig. 1.b shows the case of a key request of user Alice and Bob connected with corresponding links LA and LB to the QBB. Here link L5 is connected in parallel to a link formed by L1 and L2 and another one by L3 and L4. Assuming a fixed cost per QKD device, one can look for an optimum in overall cost as function of the single-link distance between two nodes. For a number of advanced QKD-systems, the corresponding optimal distance turns out to be around $25 \mathrm{~km}$ for a one-dimensional chain of 
links as well as for a two-dimensional network ${ }^{8}$ This is also the average distance in the SECOQC Demonstrator.

- In the case of an intruder that causes one link to fail due to any attack (e.g. L5 in the upper example), the requested key could still be delivered by other remaining routes through the network.

- Furthermore, with the same argument, if one link is corrupted by the powerful denial-of-service attack, the pre-shared initial secrets of the link under attack will run out. In a single-link scenario, human interaction is needed to restore the pre-shared secret. In contrast, in a quantum network, this key can be restored over the remaining quantum links.

\section{Network Demonstration in Vienna 2008}

To introduce and demonstrate new network functionalities and to show the various advantages over single links, we implement a universal rectangular building block (Fig. 2) out of four stations in Vienna (SIE, ERD, GUD, BREIT) and extend it by one node at a nearby city St. Pölten. All QBB-Links are implemented including the two diagonals and two short QKD-connections towards the end-users.

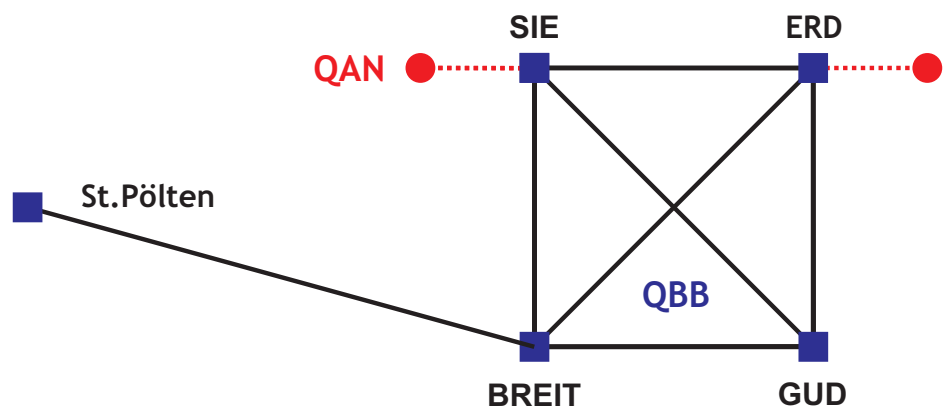

Fig. 2. (Color online). Concept of the deployed universal building block for quantum key distribution networking. The names refer to the stations of the ring network.

In contrast to the architecture of the DARPA quantum network, $\frac{5}{5}$ in which the quantum channel was actively controlled by a fiber-optical switch depending on the communication partners, in the presented SECOQC quantum network each pair of QKD-devices forming a QKD-Link remain together. The task of each QBB-Link is to grow as much key as possible and to hand it over to the network node, no matter which end-user will request for it afterwards.

All of the used QKD devices have reached high maturity before leaving the various labs and are being prepared for deployment and operation at field conditions. The feasibility of the discussed network architecture will then be tested in real-life conditions. 


\subsection{Telecom fiber ring network from Siemens}

All the links will be operated on a typical ring-shaped network to connect officebuildings of SIEMENS Austria. Fig. 3 shows the fiber ring with a circumference of approximately $63 \mathrm{~km}$. We use four node-stations distributed well over the whole city of Vienna and one in St. Poelten, another city connected by an $85 \mathrm{~km}$ long fiber. The fibers for both cross-connections are also physically deployed in the ring (connecting the two pairs of non-neighboring nodes respectively).

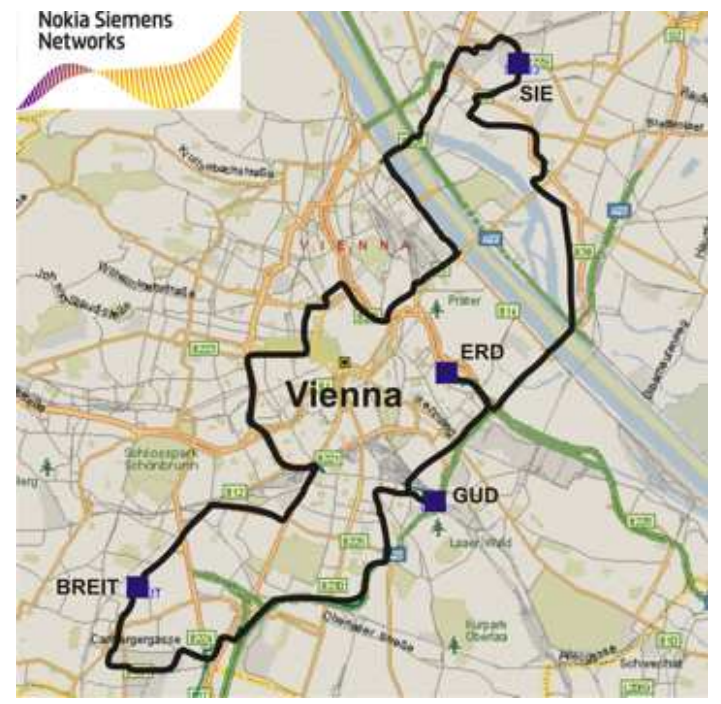

Fig. 3. (Color online). City map of Vienna including the fiber ring network and the stations SIE, ERD, GUD and BREIT. Another station, St. Pölten, is located far outside of Vienna.

Each QKD-device pair is connected by their own dark fiber serving as an exclusive quantum channel. The classical communication is managed by dedicated node modules using one additional fiber-loop in the ring, which is realized by a set of communication lasers multiplexed using CWDM spacing.

\subsection{QKD-link-devices for the Quantum Back Bone $Q B B$}

For a convincing demonstration of the network features, SECOQC has decided to develop high-rate stable QKD-Link devices. Only devices with a secure key rate of more than $1 \mathrm{kbit} / \mathrm{s}$ after authentication over $25 \mathrm{~km}$ of fibers are accepted for the stage of deployment. Clearly the link devices must fulfill tight security standards. 3 Moreover, the devices must be autonomously delivering the key for more than 6 months without human interaction. The latency time for a new start (for example, after change of the fiber connection) is limited to 1 minute.

For the stage of deployment the following systems have met these criteria and form the QBB-network: 
- IdQuantique (Switzerland): The Swiss company delivers three devices-pairs usually referred to as "Plug \& Play" system ${ }^{9}$ By attenuating a strong pulse to singlephoton level within one round-trip, no additional compensation of the drifts from the interferometers and fiber are needed.

- GAP Geneva/IdQuantique/ARC (Switzerland/Austria): The university group headed by Prof. Gisin develops one QKD system in which the position of nonempty time slots of the attenuated light pulses carries the transmitted bit value 10 Coherence between the photons reveals any intruder who would try extracting information out of the system.

- Toshiba (UK): The group of Andrew Shield develops one QKD system with two interferometers, which are stabilized by classical pulses sent after the quantum signals (weak coherent pulses approximating single photons). BB84 in the phase modulation version is implemented ${ }^{11}$ To beat the photon number splitting attack for weak coherent pulses, i.e increase the secure key rate at $25 \mathrm{~km}$, decoy states are implemented.

- University of Vienna/ARC/KTH (Austria/Sweden): Entangled photons have been studied by Prof. Zeilinger for a long time and are now being also transferred to telecommunication wavelength. Implemented this fundamental quantummechanical property by the BBM92 protocol, the correlations of detections of entangled photon-pairs at different QKD-devices are used to generate the key 12

- CNRS/Thales/ULB (France/Belgium): The consortium lead by Prof. Grangier develops the only continuous variable QKD system in the prototype. Stronger pulses with tens of photons in each pulse are used instead of weak coherent pulses. Homodyne detection replaces in this case the usage of single photon detectors.13

\subsection{QKD-link-devices for the Quantum Access Network (QAN)}

In order to connect an end-user device to the QBB, a single link towards one QBBnode must be established, whereby the shortest connection is aspired. For this quantum access network any kind of QKD devices can be employed in principle. The secure key rate to be generated for each user is expected to be far below the typical connection load at the QBB. Therefore QAN-Links call for simple devices with lower rates, but also of lower cost. As a special case, to extend QKD-networks to areas with lower penetration of deployed fiber infrastructure, free space QKD is an appropriate alternative. For this reason, two free-space QKD systems have been included:

- Ludwig-Maximilian-University Munich (Germany): The free-space system developed by the group Prof. Weinfurter has a typical working distance of $500 \mathrm{~m} .14$ To reach typical MAN-like distances the possible distance can be extended to 3 $\mathrm{km}$. Up to now, only night-time operation is possible, but the used single-photon detectors based on Silicon permit higher key generation rates.

- University of Bristol (UK): The team under Prof. Rarity developed a portable

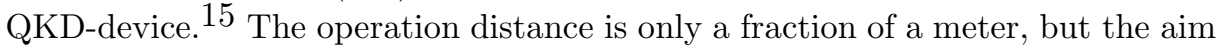


of this unique development is to enhance the security of existing applications like the interaction with ATMs by creating hand-held consumer devices for PIN protection and authentication.

\subsection{Network nodes}

All end-devices of the QBB-Links and at least one device of each QAN-Link are situated within a network node (Alice 1, Alice 2 and Alice 3 in Fig. 4) communicate with the node module. As mentioned before, each pair of QKD-devices form a link and will remain together in this presented network architecture. We do not implement switching on the level of QKD-Link devices, because one goal was to allow connectivity to the network for a broad range of heterogeneous QKD-devices only by adopting common interfaces and protocols to the network node-module.

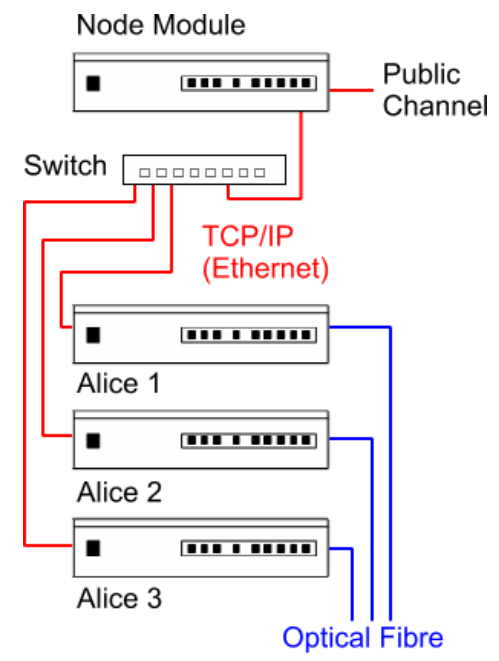

Fig. 4. (Color online). The node module manages the secret keys and the classical communication generated by all QKD devices. Device independent operation is ensured by a common interface. The needed authentication of messages is also provided by the node module.

The task of each QBB-Link is to continuously grow as much key as possible and to hand it over to the network nodes. Thereby a node module overtakes the important task of storage and handling of the generated keys. On one hand, the node module oversees the separate link-devices in the node, and even more importantly in the presented architecture, it provides them with classical communication connectivity to the partner devices of the respective QKD-Link. The indispensable message authentication of the classical part of the QKD-Link communication needed to overcome potential man-in-the-middle attacks is also provided by the node module, the QKD-protocol stack of each link being responsible to decide which communication messages are to be authenticated. 
On the other hand, the node module is further responsible for the overall network functionality by executing the QKD higher-level network protocol stack (discussed in more detail below), which in charge of routing and transporting the generated secrets on the network level. The node-module functionality implies that all nodemodules are connected over public channels with each other (Fig. 5).

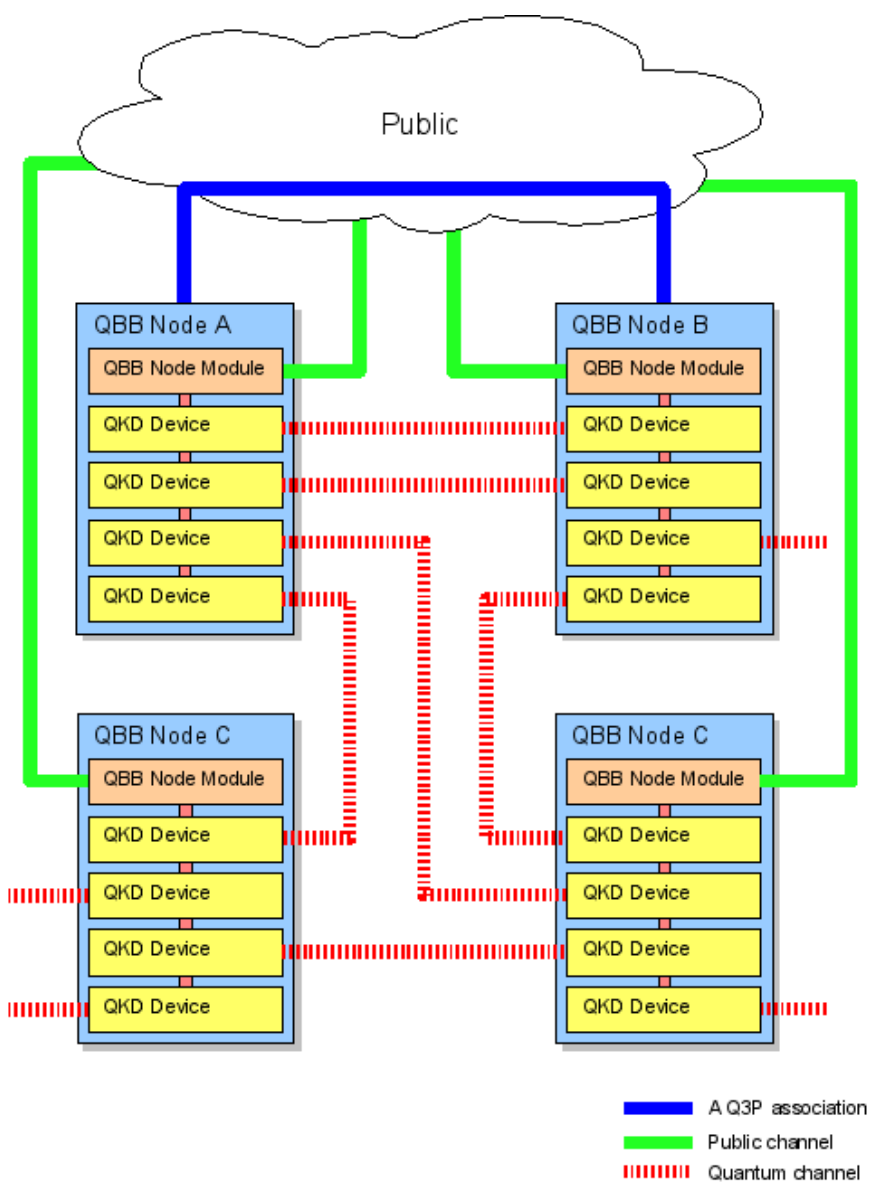

Fig. 5. (Color online). Four nodes of the QBB are connected with quantum channels and communicate over the public channels. Between nodes A and B, one specific connection is highlighted to emphasize the communication of the Q3P network protocol.

\section{Network Protocols}

As already mentioned, several different devices will compose the SECOQC QBB network. As each of them has different characteristics and interfaces, a common protocol has been designed to access services provided by the devices in an uniform manner: the Quantum-Point-to-Point-Protocol or Q3P 16 
This protocol serves as Point-to-Point Protocol between a pair of QBB nodes (see Fig. 5) which enables the devices underneath to carry out the classical communication protocol for key distillation over an authenticated channel, while simultaneously providing authentication and encryption for upper layers in the network. Both authentication and encryption are information-theoretically secure.

Using Q3P as an uniform building block interconnecting a pair of QKD devices one can now employ traditional network protocols on top of it. However most widespread protocols like TCP/IP are not directly compatible with the specific requirements of quantum key distribution: network reliability in terms of TCP-like re-sending packets has to be considered carefully since the content of the packets in a QKD case is highly sensitive with respect to confidentiality and/or authenticity.

Therefore well known protocols have been adapted to form a new set of endto-end networking mechanisms $\frac{17}{17}$ The QKD Routing Layer (QKD-RL) Protocol addresses the routing information within the QBB nodes. This protocol follows the pattern of OSPF but includes essential modifications to address the specific requirements arising from the sensitivity and scarceness of key material.

Finally, the QKD Transport Layer (QKD-TL) Protocol adopts TCP/IP and introduces new approaches to dealing with highly congested networks based on quantum key resources. This protocol finally lets users exchange information across the network with perfect confidentiality and authenticity on an end-to-end basis.

All three protocols sketched above are independently designed and each presents a standard-like interface, which can easily be introduced in current telecom network infrastructures. No applications running on upper layers need to be modified in order to use the unconditionally secure key material.

\section{Conclusions and Outlook}

A quantum key distribution network covering a full inner-city metropolitan area network using seven fiber-based QKD devices, realized by five different working principles and two free-space QKD setups will be demonstrated in September 2008 in Vienna. This implementation in the framework of the EU-funded FP6project SECOQC clearly shows the feasibility of constructing highly integrated QKD-networks. Heterogeneous modern QKD devices are combined through common interfaces and universal node modules into unified secret distribution infrastructure. This important step towards practical deployment offers end-users and network providers a vision of future secure communication facilities.

\section{Acknowledgments}

This work was supported by the European Commission through the integrated project SECOQC (Contract No. IST-2003-506813). 


\section{References}

1. D. Gottesman et al., Security of quantum key distribution with imperfect devices, Quant. Inf. Comput. 5 (2004) 325-360.

2. M. Dušek et al., Quantum Cryptography, Progress in Optics, Vol. 49, ed. E. Wolf (Elsevier, 2006), 381-454.

3. V.Scarani et al., A Framework for Practical Quantum Cryptography. To be published (2008).

4. N. Gisin et al., Quantum cryptography, Rev. Mod. Phys. 74 (2001) 145-195.

5. C. Elliott et al., Quantum Cryptography in Practice, Comput. Commun. Rev. 3 (2003) 227-238.

6. R. Alléaume et al., SECOQC White Paper on Quantum Key Distribution and Cryptography, quant-ph/0701168

7. M. Peev et al., A Quantum Key Distribution Network: Integrated Design and Prototypical Implementation, in European Conference on Lasers and Electro-Optics, OSA

Technical Digest Series (Optical Society of America, Washington D.C., 2007); CLEO Europe, Paper JSI3-5-THU, Munich, Germany (2007).

8. R. Alléaume et al., Long-distance quantum key distribution networks: cost calculations and optimal working points of individual links, in preparation (2008).

9. A. Muller et al., "Plug and Play" systems for quantum cryptography, Appl. Phys. Lett. 70 (1997) 793-795.

10. D. Stucki et al., Fast and simple one-way quantum key distribution, Appl. Phys. Lett. 87 (2005) 194108.

11. J. F. Dynes et al., Practical quantum key distribution over 60 hours at an optical fiber distance of $20 \mathrm{~km}$ using weak and vacuum decoy pulses for enhanced security, Opt. Express 15 (2007) 8465-8471.

12. H. Hübel et al., High-fidelity transmission of polarization encoded qubits from an entangled source over $100 \mathrm{~km}$ of fiber, Opt. Express 15 (2007) 7853-7862.

13. J. Lodewyck et al., Quantum key distribution over $25 \mathrm{~km}$ with an all-fiber continuousvariable system, Phys. Rev. A 76 (2007) 042305.

14. T. Schmitt-Manderbach et al., Practical free space quantum cryptography, in Quantum Electronics Conference EQEC '05 (2005) 305.

15. J. L. Duligall et al., Low cost and compact quantum key distribution, New J. Phys. 8 (2006) 249.

16. O. Maurhart, Q3P: A Proposal, SECOQC deliverable (2006), unpublished.

17. M. Dianati et al., Architecture and Protocols of the Future European Quantum Key Distribution Network, to appear in Security and Communication Networks 1 (2008). 